\title{
Penetrating Trauma to the Mediastinal Vessels: a Taxing Injury
}

\author{
Pradeep H. Navsaria ${ }^{1}$ - Sharfuddin Chowdhury ${ }^{1} \cdot$ Andrew J. Nicol $^{1} \cdot$ Sorin Edu $^{1}$. \\ Nadraj Naidoo ${ }^{1,2}$
}

Published online: 23 January 2016

(C) Springer International Publishing AG 2016

\begin{abstract}
More than $90 \%$ of thoracic great vessel injuries are due to penetrating trauma. The increased incidence of civilian penetrating (stab and low-velocity gunshot wounds) chest trauma and improved emergency medical services (EMS) have stemmed in an increasing number of seriously injured, but potentially salvageable patients, presenting to trauma centers. Penetrating mediastinal vascular injuries are associated with a high mortality. Unstable patients present a diagnostic and operative challenge to the surgeon, and require damage control resuscitation (DCR) and immediate surgery. DCR begins with the restrictive fluid administration, permissive hypotension, early blood product therapy that includes initiation of massive transfusion protocol (MTP), temporary hemostasis by balloon tamponade, tube thoracostomy, and/or
\end{abstract}

This article is part of the Topical Collection on Penetrating Injuries To Major Vessels

Pradeep H. Navsaria

pradeep.navsaria@uct.ac.za

Sharfuddin Chowdhury

dr_smahmud@yahoo.com

Andrew J. Nicol

andrew.nicol@uct.ac.za

Sorin Edu

sorinedu@yahoo.com

Nadraj Naidoo

nadraj.naidoo@uct.ac.za

1 Trauma Center, Groote Schuur Hospital, University of Cape Town, Anzio Road, Observatory, Cape Town 7925, South Africa

2 Department of Vascular Surgery, Groote Schuur Hospital, University of Cape Town, Anzio Road, Observatory, Cape Town 7925, South Africa resuscitative thoracotomy. Stable patients can undergo a rapid and aggressive workup with screening computerized tomographic angiography (CTA) followed by catheter angiography for unclear CTA findings, and when feasible, endovascular intervention.

Keywords Penetrating thoracic trauma $\cdot$ Mediastinal vessels · Damage control resuscitation

\section{Introduction}

All thoracic injuries, due to both blunt and penetrating mechanisms account for $20-25 \%$ of traumatic deaths [1]. Trauma to the mediastinal vascular structures includes the aorta (ascending, arch and its branches, and descending aorta), the pulmonary vessels, the intrathoracic vena cava, and the innominate and azygos veins. Reports of penetrating trauma to the mediastinal vessels are lacking in the literature until the twentieth century due to the absence of survivors. More than $90 \%$ of thoracic great vessel injuries are due to penetrating trauma [2]. The increased incidence of civilian penetrating (stab and low-velocity gunshot wounds) chest trauma and improved emergency medical services (EMS) have stemmed in an increasing number of seriously injured, but potentially salvageable patients, presenting to trauma centers. Strategies for treating thoracic injuries were established only after the Second World War. Current knowledge regarding treatment of injured thoracic great vessels has been derived primarily from experience with civilian injuries. Great vessels injuries have been repaired with increasing frequency, a phenomenon that has paralleled the development of techniques for elective surgery of the thoracic aorta and its major branches. The management of patients with penetrating thoracic trauma is determined by the patient's hemodynamic status. 


\section{Penetrating Trauma to the Mediastinal Vessels}

The presence of a mediastinal vessel injury following penetrating trauma can be obvious or subtle in presentation. It depends on multiple factors such as the mechanism, length of the sharp object, type of firearm, distance of the patient from the firearm, and the vessel involved. The physician should be aware of the possibility of mediastinal vascular injury in case of transmediastinal gunshot wound or presence of a wound at the root of the neck. The patient may present as pulseless or moribund. The diagnosis is usually made during the resuscitative thoracotomy or urgent thoracotomy. In a stable patient who becomes stable after resuscitation, the diagnosis can be made with computed tomographic angiogram or catheter angiography in selected patients. Patients may present with a mediastinal hematoma, massive hemothorax, cardiac tamponade, or external bleeding. Hypotension, upper extremity hypertension, unequal blood pressures or pulse discrepancy in the affected vessel's distribution, a thrill or bruit at the root of the neck, expanding hematoma at the thoracic outlet, intra-scapular murmur, brachial plexus injuries, and stroke or coma also support the diagnosis of a great vessel injury [3].

\section{Approach to Mediastinal Vascular Injury: Hemodynamic Abnormal}

On presentation to the emergency department, all patients with possible mediastinal vascular trauma should undergo a rapid evaluation according to the Advanced Trauma Life Support (ATLS) guidelines. Hemodynamically unstable patients require immediate damage control resuscitation (DCR) followed by mandatory emergency surgery for nonresponders. For penetrating major vascular injuries, DCR begins with the restrictive clear fluid administration, permissive hypotension, early blood product therapy that includes initiation of massive transfusion protocol (MTP), temporary hemostasis by balloon tamponade, tube thoracostomy, and/or resuscitative thoracotomy. The aim is to prevent or arrest the lethal triad of acidosis, hypothermia, and coagulopathy.

\section{Restrictive Fluid Administration}

Fluid administration for resuscitation of trauma patients presenting with hemorrhagic shock remains controversial. There is a recent paradigm shift of the historical practice of overzealous administration of clear fluids, to restrict fluid. Massive crystalloid and colloid fluid resuscitation result in dilutional coagulopathy, a hyperbolic systemic inflammatory response syndrome (SIRS), pulmonary edema, an increased incidence of adult respiratory distress syndrome (ARDS), polycompartment syndrome, electrolyte imbalance, and worse overall survival [4-9]. In a recent double-blind, randomized controlled trial, it was demonstrated that in penetrating trauma, resuscitation with colloids (hydroxyethyl starch 130/ 0.4 ) has a better effect on the renal outcome and faster lactate clearance without clinically relevant coagulopathy compared with crystalloids (0.9\% saline) [10].

\section{Permissive Hypotension}

There is now more evidence available in support of permissive hypotension in resuscitating bleeding trauma patients. The current trend is to limit fluid administration, targeting a lower than the normal systolic blood pressure (SBP) of $80-$ $90 \mathrm{mmHg}$, or mean arterial pressure (MAP) of $50 \mathrm{mmHg}$ until surgical control of the bleeding is achieved. Evolving evidence suggests that overzealous fluid administration before hemostasis leads to further bleeding through hydraulic acceleration of hemorrhage, soft clot dissolution by raising intravascular pressure and dilution of clotting factors [11].

\section{Blood Product Therapy}

The administration in a 1:1:1 ratio of packed red blood cells, fresh-frozen plasma, and platelets (of individual units) is related with improved survival [12•]. Adjunctive treatment with recombinant factor VIIa, cryoprecipitate, and tranexamic acid $[13,14 \cdot]$ may improve coagulopathy. Additional benefits of earlier administration of blood products during the resuscitation phase include a substantial reduction of the volume of crystalloid fluid requirement during resuscitation $[15,16]$, improved overall efficiency, decreased total blood product use during a patient's hospital stay, and economic savings [12•, $17]$.

\section{Balloon Tamponade}

Balloon tamponade by Foley catheter (FC) to achieve temporary control of bleeding vessels is a useful adjunct to damage control resuscitation. It is a simple technique of placing of a FC into a hemorrhaging wound, inflating the balloon with either water or saline in an attempt to achieve hemostasis by compression of the injured vessels. This simple technique does not require any expertise or specialized equipment. It can temporarily arrest hemorrhage allowing for patient interhospital transfer, and further investigation to allow for diagnosis and planning of definitive surgery [18]. Although Foley catheter balloon tamponade (FCBT) is mostly described to control bleeding in the neck [19•], it is also useful for the wound at the junction of the neck and thoracic inlet, peri clavicular, or sternoclavicular regions (Fig. 1).

\section{Tube Thoracostomy}

Massive hemothorax following mediastinal vascular injury can lead to cardiopulmonary collapse. Immediate chest 
Fig. 1 a Successful Foley catheter balloon tamponade in a patient with a right lateral infraclavicular stab wound (white arrow). b Catheter angiography showing a proximal right subclavian artery injury that was approached through a median sternotomy with the right supraclavicular neck extension (black arrow)
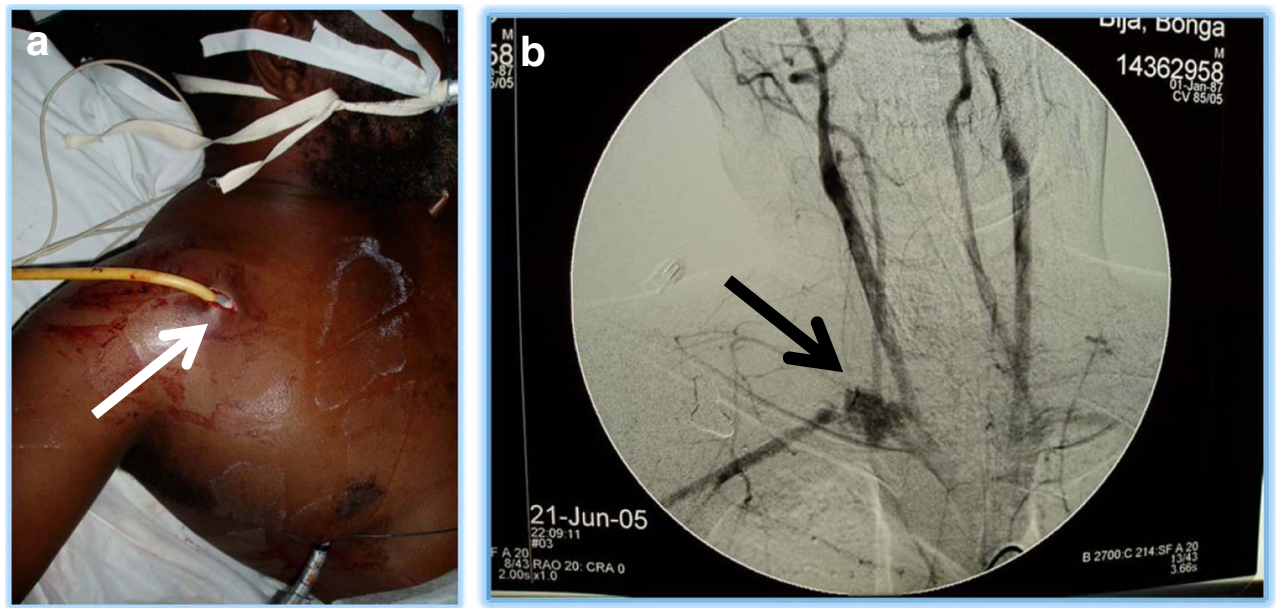

decompression by tube thoracostomy is an essential part of DCR. Unstable patients with penetrating thoracic trauma should undergo immediate bilateral tube thoracostomy. The chest tube can be connected to a cell saver device for autotransfusion. Immediate drainage of a large volume of blood (more than $1.5 \mathrm{~L}$ ) or ongoing bleeding (200-250 mL/h) may indicate major vascular injury and mandates the need for immediate thoracotomy.

\section{Resuscitative Thoracotomy}

Instant control of bleeding by emergency room thoracotomy is a part of DCR before definitive surgery. It allows the release of cardiac tamponade and arrest of bleeding from cardiac, lung, and great vessel injury and subsequently improves cardiac output [20]. It also enables resuscitation through open cardiac massage, defibrillation with internal paddles, and intracardiac administration of drugs. Cross-clamping of descending thoracic aorta facilitates cerebral and coronary perfusion by diverting blood flow as well as slows down distal bleeding from combined intra-abdominal or pelvic injury. If acted timely, resuscitative thoracotomy (RT) can be lifesaving in carefully selected patients. Survival may reach up to $40 \%$ of trauma victims with penetrating thoracic wounds and cardiac tamponade [21]. Patients with isolated penetrating chest injuries have the greatest chance of survival than the patients with multi-cavity injuries $[22,23]$. When RT is performed, survival is higher with stab wounds (18-24\%) than gunshot wounds (4-5\%) [24-28]. The physiologic state just before surgery is commonly not described in the literature, and different vague terms such as "no signs of life," "no vital signs," "lifeless," and "agonal" are frequently utilized. A recent survey reported a lack of agreement regarding the indications for RT as well as in defining "signs of life" [29, 30•]. The high-quality prospective studies are also absent due to nature and ethical issues involving the care of dying trauma victims [30•]. Despite the limitations, RT is supported in patients with penetrating thoracic injury who present to the emergency room (ER) with any sign of life (SOL) or deteriorate shortly after arrival [21, $31,32 \bullet \cdot$. The available data also support refraining from RT in both penetrating and blunt trauma patients who never had witnessed SOL $[31,32 \bullet \cdot]$. Thus, most of the debate regarding the indications for RT (Table 1) is focused on patients who develop cardiopulmonary arrest before arrival to the hospital.

For RT, a standard incision is a left anterolateral thoracotomy that extends from the costal margin to the mid-axillary line in the fifth intercostal space, avoiding injury to the internal mammary artery and intercostal vessels by incising the skin, subcutaneous tissue, pectoralis major, and the intercostals down to the pleura. Incision of the pleura and use a Mayo scissors to divide the remaining muscle and pleura avoid injury to the underlying lung. Insertion of a large Finochietto retractor is performed to expose thoracic cavity. If the entrance wound is on the right side of the chest, surgeons consider a right anterolateral thoracotomy. If required, additional exposure may be gained by dividing the sternum transversely with a Lebske knife or trauma shears, or doing a clamshell thoracotomy. After evacuating blood from the chest, attention is directed to the injury. If a great vessel is injured and bleeding, temporary control can be gained by direct digital pressure,

Table 1 Indications for resuscitative thoracotomy in penetrating chest injuries

Asystole with signs of life in the preceding five minutes

Pulseless electrical activity on ECG monitors with cardiac arrest

In-hospital hypovolemic cardiac arrest

A hypotensive patient with a precordial injury, who has a systolic blood pressure of less than $70 \mathrm{mmHg}$, is not responding to fluid resuscitation

Drainage of $>1.51$ of blood on chest drain insertion

Continuous bleeding $>200 \mathrm{ml}$ per hour into chest drain

Cardiac tamponade

Thoracic great vessels injury

Loss of thoracic wall 
occlusion with a Satinsky clamp, or even by insertion and inflation of a Foley catheter balloon into the wound, and held under tension. If hemopericardium is present, the pericardium may be opened with a vertical incision after careful identification and preservation of phrenic nerve. Once the hematoma is evacuated, the heart is delivered from the pericardial sac, and hemorrhage can be controlled by applying digital pressure, suture, or staples. Foley catheter balloon is also useful for temporary control. If air embolism is suspected, the pulmonary hilum is clamped or the affected lung may be twisted $180^{\circ}$ and air in the aorta evacuated. After the cause is addressed, a cross-clamp can be applied to the descending thoracic aorta after sweeping the lung anteromedially and exposing the posterior mediastinum. Temporary arresting mechanical ventilation and placement of a nasogastric tube are helpful adjuncts to visualize and localize a flaccid descending aorta. The intravascular volume is restored, and if the patient responds, he or she is transported to the operating room for definitive repair.

\section{Approach to Mediastinal Vascular Injury: Hemodynamic Normal}

Patients who are stabilized after resuscitation or present hemodynamically normal must be carefully monitored to detect early decompensation. Patients may appear stable following a transmediastinal gunshot wound, even when they have lifethreatening injuries [33]. Penetrating transmediastinal injury is defined as evidence of a single missile entry and exit on opposite sides of the thorax, missile entry and missile retention on opposite sides of the thorax, and missile entry on one side of the thorax with missile retention in the mediastinum [34, 35].

\section{Investigational Workup for Stable Patients}

\section{Chest Radiograph}

All patients with suspected mediastinal vascular injury should have a chest radiograph or a Lodox Statscan ${ }^{\text {TM }}$ (low-dose full-body digital X-ray). It is very useful to place radiopaque markers to identify the entrance and exit sites. Radiographic findings that may suggest a mediastinal vascular injury include abnormal central mediastinal opacities, massive hemothorax, foreign bodies (shrapnel or bullets), or missile trajectories close to the great vessels (Table 2). A trajectory with a confusing course may indicate a migrating intrapleural or, intravascular or intracardiac bullet, suggesting distal embolization.
Table 2 Chest radiographic features of suggestive of mediastinal vascular injury

Massive left hemothorax

Apical pleural hematoma

Any abnormal opacity in the superior mediastinum

Obliteration or double shadow of aortic knob contour

Loss of perivertebral pleural stripe

Lateral displacement of trachea

Loss of aortopulmonary window

\section{Computerized Tomographic Angiography}

A standard supine chest radiograph does not provide diagnostic sensitivity to rule out a vascular injury. Many authors recommend multidetector computed tomography (CT) of the chest as a screening tool. The sensitivity and negative predictive values are almost $100 \%$ for mediastinal hematoma usually associated with aortic disruption [36-38]. Computerized tomographic angiography (CTA) is safe and reduces the need for an invasive diagnostic procedure of selected patients with transmediastinal gunshot wounds [39]. It helps to define the trajectory relative to vascular and aerodigestive structures within the mediastinum and thoracic inlet [40]. Many surgeons use CT findings alone as a roadmap to diagnose an injury and unusual vascular anomalies to plan surgery. Motion artifact in the proximal ascending aorta can be difficult to interpret on CT. In this situation, a catheter angiography is indicated.

\section{Transesophageal Echocardiogram}

Multiplanar transesophageal echocardiogram (TEE) images the thoracic aorta consistently. Although the accuracy of TEE is operator-dependent, the sensitivity and specificity of TEE in the diagnosis of mediastinal aortic injury is reported as high as $100 \%[41,42]$. The distal ascending aorta and proximal aortic arch are often obscured by tracheobronchial air artifact. Images can be unreliable for this segment. Moreover, the presence of atheromatous disease or pneumomediastinum may confound the detection of aortic injury. For these reasons, several authors do not advocate TEE in the acute setting especially in polytrauma patients [3]. TEE is portable, and the main advantage is it allows on-table evaluation of unstable patients [43].

\section{Intravascular Ultrasonography}

Intravascular ultrasonography (IVUS) is a relatively modern diagnostic tool to detect vascular injury. It is an expressly designed catheter with a high-frequency miniature ultrasound transducer attached to the distal end. The proximal end is 
connected to the computerized ultrasound equipment. It can obtain real-time $360^{\circ}$ images of the aorta. It is performed by introducing the ultrasound probe through an arterial sheath. A recent study found that IVUS accomplished better than catheter angiography in patients who had equivocal CTAs [44]. At present, the lack of a reference standard procedure, the high cost of the disposable transducers, and its invasive nature limit the use of IVUS in the trauma setting.

\section{Catheter Angiography}

In penetrating thoracic trauma, catheter angiogram is indicated in suspected mediastinal vascular injuries including aortic, innominate, carotid, or subclavian arterial injuries. For intraoperative proximal and distal control of these vessels, different incisions are required. Arteriography helps in localizing the injury and planning the appropriate incision. The catheter angiography is not always above limitations. Temporarily sealed off laceration or if the column of aortic contrast overlies a small area of extravasation may give a false impression of negative aortogram. Obtaining tangential views to possible injuries may help to overcome this limitation. An added advantage of catheter angiography is therapeutic endovascular intervention.

\section{Surgical Approach to Mediastinal Vessels}

Penetrating injuries to the mediastinum usually have a high probability of injury to the vascular and other vital structures. Mandatory exploration was the practice in the past. With the evolution of modern diagnostic and radiologic investigations including combined arteriography, esophagoscopy, bronchoscopy, and echocardiography help to decrease unnecessary explorations in hemodynamically stable patients. A CT angiogram of the chest often shows the bullet trajectory as well as any injury to the vascular structures and dictates the need for surgery.

A detailed understanding of the normal and variant anatomy and structural relationships of mediastinal vessels is vital for the surgeon. Thoracic arch anomalies are relatively common. Venous anomalies are infrequent with the most common being absence of the left innominate vein and persistent left superior vena cava. Knowledge of such anomalies is essential for both open and catheter-based therapies.

When the decision is made to operate on a patient with mediastinal vascular injury, a median sternotomy is standard to access the superior mediastinum. A large red jelly mediastinal hematoma usually lies above the pericardium obscuring the anatomy. It signifies the major vascular injury, and careful dissection is necessary to avoid major trouble. The pericardium is the anatomical barrier that prevents extension of the mediastinal hematoma. So in a hostile environment, it is a useful trick to open the pericardium and follow the aortic arch upward into the hematoma to identify the vessels of the superior mediastinum. After identifying the left innominate vein, the gatekeeper of the mediastinum, dividing and ligating it opens up the superior mediastinum. It gives access to the superior aspect of the aortic arch and its branches. If the thymus is on the way, it can be divided between clamps and ligated. The next step is to identify the bifurcation of the innominate artery and the right vagus nerve that crosses in front of the proximal right subclavian artery and to secure it to prevent an iatrogenic injury. In the superior mediastinum, veins are lying superficially, and the arteries are deep. Venous injury can be controlled by a side-biting clamp and simple lateral repair. If the repair is impossible, division and ligation can be done without any hesitation. Then, the aim is to achieve proximal control by exposing the ascending aorta inside the pericardium. The distal control can be obtained by putting a clamp on the distal innominate, right subclavian, right carotid and proximal descending arteries [45].

\section{Ascending Aortic Injury}

The ascending aortic injury is relatively uncommon. In patients who reach to a trauma center with stable hemodynamics, the survival rate is almost $50 \%$ [46]. To gain access to the aortic arch, an extension of sternotomy wound to the neck is necessary $[47 \bullet \bullet]$. Hemorrhage may limit the exposure. Temporary balloon tamponade can be useful in this situation. Lateral aortorrhaphy for simple anterior wall laceration and additional posterior wall injury makes repair difficult. In this case, cardiopulmonary bypass may be necessary.

\section{Innominate Arterial Injury}

Right cervical extension of a median sternotomy is often essential for dealing with the innominate artery. In this case, division of the strap muscles down low, near the insertion into the sternum, to expose the carotid sheath is necessary. Simple repair is often impossible in this area. Bypass exclusion technique from the ascending aorta to the distal brachiocephalic trunk, just proximal to the bifurcation, is a feasible option (Fig. 2) [48]. The injured area is escaped until the bypass is performed. A $10-\mathrm{mm}$ dacron graft is sewn end to side to the ascending aorta with a partial occluding clamp proximal to the hematoma. The distal innominate artery is isolated proximal to its bifurcation. This is divided and sewn end to end to the graft without systemic anticoagulation, hypothermia, shunts, or cardiopulmonary bypass. After the restoration of flow, the area of hematoma is controlled with a large partial occluding clamp, and the aortic arch is oversewn. The innominate vein may be ligated if concomitantly injured or previously divided [3]. 

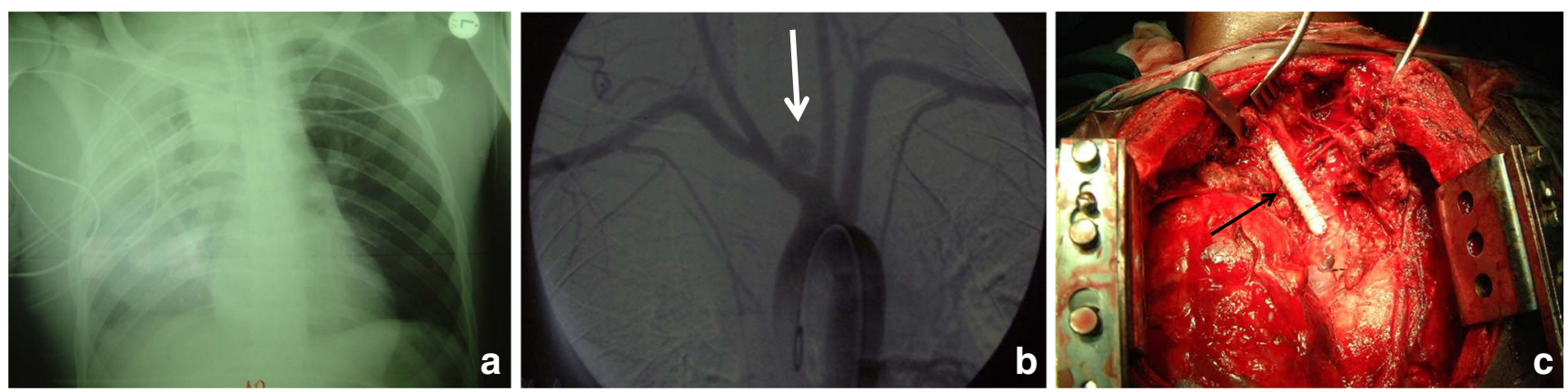

Fig. 2 a Admitting chest radiograph showing massive right haemopneumothorax with abnormal mediastinal opacity following stab wound. b Catheter angiography revealing innominate artery false aneurysm (white arrow). c Intraoperative repair with an interposition PTFE graft (black arrow)

\section{Proximal Left Common Carotid Artery Injury}

Injury to the left proximal common carotid artery is also approached in a similar fashion to an innominate artery injury through a median sternotomy with left cervical extension. Care must be taken to identify and preserve the left vagus nerve as it descends between the carotid and left subclavian arteries to cross in front of the aortic arch and give off the left recurrent laryngeal nerve. Initial attempts to partially occlude the aorta with side-biting (partially occluding) clamps may afford control of the origin of the carotid without complete occlusion of the aorta. If this is not successful, then full clamping of the aorta may be necessary. Exposure of the right common carotid artery is carried out in a similar fashion. Management of the patient's hemodynamic parameters mandates full assistance of the anesthesiologist because of the profound increase in afterload resistance that will develop. Vascular clamp time should be kept to a minimum and should be restricted to within a 20-30-minute period. Bypass graft repair is ideal over end-to-end anastomosis for injury at the origin.

\section{Proximal Subclavian Vessels Injury}

To approach the right subclavian vessels in the mediastinum or at the thoracic outlet, a median sternotomy with cervical extension is employed. High left anterolateral thoracotomy (second or third intercostal space) is for the proximal control of the left-sided subclavian artery injury. A separate left supraclavicular incision may be necessary for distal control. For exposure, the clavicular head of the sternocleidomastoid and the omohyoid are divided close to the clavicle. After retraction of the internal jugular vein medially, the subclavian vein can be accessed. The phrenic nerve lying on the scalene fat pad should be identified and preserved at any cost. The subclavian artery is lying deep to the anterior scalene muscle. To get access to the subclavian arteries, piecemeal division of the muscle with scissors is recommended to avoid injury to the brachial plexus. On the left side, the thoracic duct enters at the junction of the left subclavian and internal jugular veins. If it is injured, it needs suture ligation otherwise it can be left alone [45]. Associated brachial plexus injury is also common in subclavian arterial injuries. Documentation of preoperative neurologic status is therefore vital.

\section{The Descending Thoracic Aorta Injury}

Posterolateral thoracotomy via the fourth intercostal space is for the injury to the descending thoracic aorta. Proximal control can be achieved by encircling the vascular tape between left subclavian and left common carotid artery after exposing the transverse aortic arch. Then, another vascular tape can be passed to surround the left subclavian artery. Careful mobilization is vital to avoid injury to the left recurrent laryngeal nerve that is often difficult to visualize in the hematoma. Currently, vascular clamping and immediate reconstruction are the standard technique to repair. Three commonly employed adjuncts to this approach include pharmacologic agent; temporary, passive bypass shunts; and a pump-assisted left heart bypass. In the latter approach, two options exist: traditional pump bypass, which requires heparin, and use of centrifugal (heparinless) pump circuits. All three of these adjuncts to the clamp-and-repair technique should be in the armamentarium of the surgeon, who must choose the approach most appropriate to the particular clinical situation [3]. Vascular clamps are applied in proximal aorta, distal aorta, and left subclavian artery. Upon entering, the hematoma back-bleeding from intercostal arteries are often encountered. Indiscriminate ligation of intercostal vessels should be avoided. Mobilization of the aorta from esophagus minimizes the risk of secondary aortoesophageal fistula. Repair of a small laceration by primary suture is performed, relatively larger one either by end-to-end anastomosis or interposition graft. Most common complication after repair of descending thoracic aortic injury is paraplegia although the development of postoperative paraplegia is multifactorial including perioperative hypotension, indiscriminate ligation of intercostal arteries, and longer clamp occlusion during repair [49]. 


\section{Internal Mammary Artery}

Injury to the internal mammary artery (IMA) can be fatal. In a young patient, blood loss can be more than $300 \mathrm{~mL} / \mathrm{min}$ following an IMA injury. They present with massive hemothorax or even cardiac tamponade. Such injuries are usually picked up during thoracotomy, and simple suture ligation is enough to control hemorrhage.

Pulmonary artery injury is typically uncommon. Mortality is as high as $70 \%$ with central pulmonary vessels injury [2]. Intrapericardial pulmonary arteries are approached via median sternotomy. The main and proximal left pulmonary arteries are exposed with minimum dissection [50, 51]. Dissection between superior vena cava and ascending aorta is necessary to expose intrapericardial right pulmonary artery. Anterior injuries can be repaired primarily while posterior injuries are difficult to repair and usually require cardiopulmonary bypass.

\section{Venous Injuries}

Conservative, nonoperative approach for isolated mediastinal venous injuries remains the standard. Patients who reach to the hospital with isolated venous injury are usually stable, unless the thoracic vena cava, pulmonary veins, or subclavian veins are involved. Isolated mediastinal superior or inferior vena cava injury is infrequently reported. Injury to this area is associated organ trauma and mortality as high as $60 \%$. Repair of intrathoracic inferior vena cava is tough and require total cardiopulmonary bypass. Superior vena cava injuries are repaired by lateral venorrhaphy. The intracaval shunt is often necessary [52]. Complex injuries are better repaired by PTFE patch or Dacron interposition tube graft than time intensive reverse saphenous vein graft. Temporary hilar occlusion may be necessary to control hemorrhage from pulmonary veins. In the event of pulmonary vein ligation, appropriate lobectomy and or pneumonectomy needs to be done. Subclavian veins are approached in a similar fashion to subclavian arteries. Bleeding is controlled by either lateral venorrhaphy where possible or ligation. Azygos vein injury is usually associated with concomitant injury to the innominate artery, trachea or bronchus, and superior vena cava and in most cases fatal. Surgical repair through median sternotomy is enormously difficult. It may even be difficult to reach through a right anterolateral thoracotomy, requiring an extension across the sternum. For successful repair, combined incisions and approaches are frequently needed and best managed by ligation [53].

\section{Damage Control Options}

Damage control options are very limited in the mediastinal vascular injuries. In extremes, ligation of the injured artery is certainly an option accepting the risk of stroke. A temporary intraluminal shunt is not resounding and has no long-term survival benefit. In isolated subclavian vessels injury, if bleeding from a missile tract, inserting a Foley catheter and inflating the balloon to stop the bleeding temporarily followed by forearm fasciotomy is an option to buy valuable time [45].

\section{Endograft Repair}

Endograft repair of mediastinal vascular injuries remains a technical challenge for the surgeons exclusively in injuries at the origin, ascending or arch of the aorta. In a stable patient, placement of the endo-aortic graft in descending thoracic aorta is rational (Fig. 3). Preoperative planning involves carefully protocolized CT angiogram defining the size, tortuosity, proximal and distal landing zone, and angulation of arterial vessels for determination of appropriateness or feasibility of introducer sheaths and devices capable of covering the aortic injury. The American Association for the Surgery of Trauma (AAST) multicenter study with its two follow-up manuscripts
Fig. 3 a Descending thoracic aortic false aneurysm following a transthoracic gunshot injury. (black arrow). b Endograft repair with successful deployment of a covered stent (black arrow)
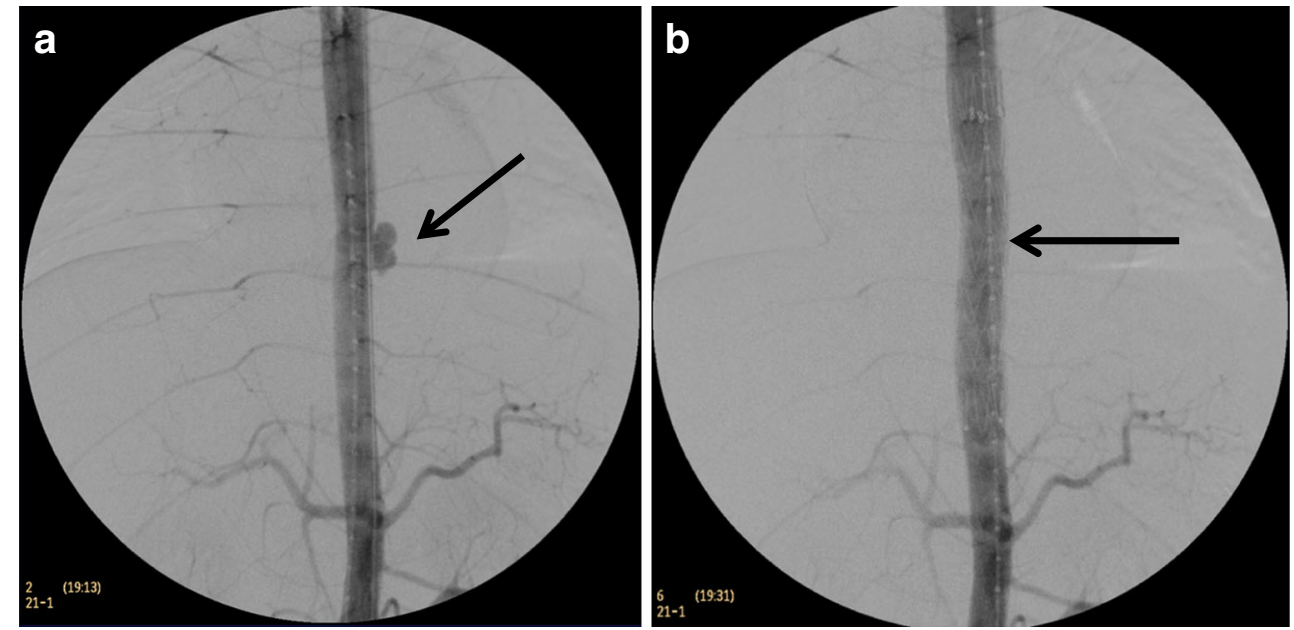
Fig. 4 Management algorithm for patients with mediastinal injuries
Stable

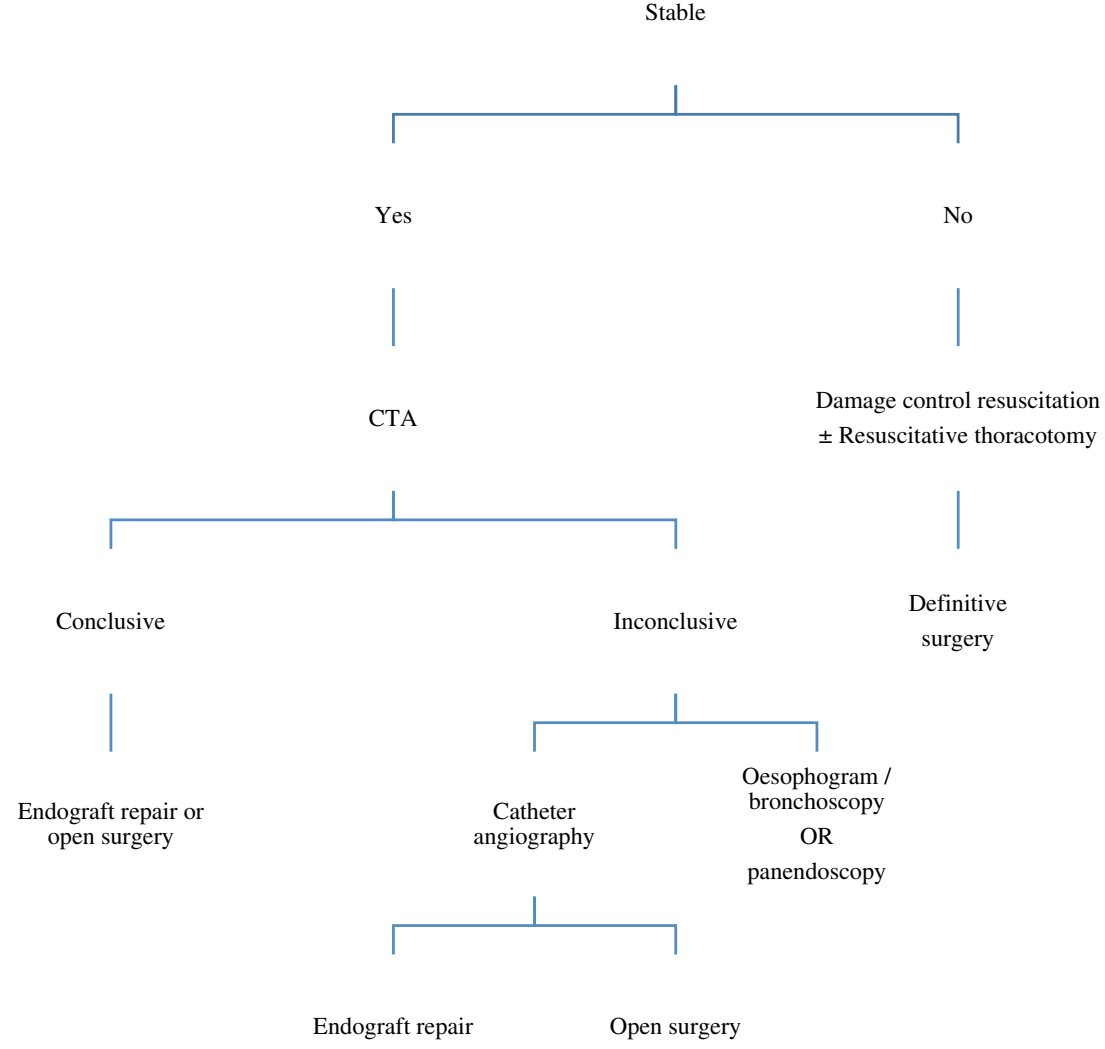

(TEG) are monitored carefully and corrected with appropriate transfusion of blood or blood component. In the case of acute kidney injury, renal replacement therapy may also require. Removal or change of lines and tubes as earliest possible is performed to reduce the source of infections. Antibiotic therapy should be reserved for established source of infection.

\section{Conclusion}

Penetrating mediastinal vascular injuries are associated with a high mortality. Unstable patients present a challenge to the surgeon and require resuscitation and immediate surgery. Stable patients can undergo a rapid and aggressive workup with screening CTA followed by catheter angiography for unclear CTA findings, and when feasible, endovascular intervention (Fig. 4).

\section{Compliance with Ethical Standards}

Conflict of Interest The authors declare that they have no competing interests.

Human and Animal Rights and Informed Consent This article does not contain any studies with human or animal subjects performed by any of the authors. 


\section{References}

Papers of particular interest, published recently, have been highlighted as:

- Of importance

•- Of major importance

1. LoCicero 3rd J, Mattox KL. Epidemiology of chest trauma. Surg Clin N Am. 1989;69(1):15-9.

2. Mattox KL, Feliciano DV, Beall Jr AC, et al. Five thousand seven hundred sixty cardiovascular injuries in 4459 patients. Epidemiologic evolution 1958-1988. Ann Surg. 1989;209:698.

3. Wall MJ, Hirshberg A, LeMaire. Thoracic aortic and thoracic vascular injuries. Surg Clin N Am. 2001;81(6):1375-93.

4. Cotton BA, Guy JS, Morris Jr JA, et al. The cellular, metabolic, and systemic consequences of aggressive fluid resuscitation strategies. Shock. 2006;26(2):115-21.

5. Daugherty EL, Liang H, Taichman D, et al. Abdominal compartment syndrome is common in medical intensive care unit patients receiving large-volume resuscitation. $\mathrm{J}$ Intensive Care Med. 2007;22(5):294-9.

6. O'Mara MS, Slater H, Goldfarb IW, et al. A prospective, randomized evaluation of intra-abdominal pressures with crystalloid and colloid resuscitation in burn patients. J Trauma. 2005;58(5):10118 .

7. Giannoudis PV, Fogerty S. Initial care of the severely injured patient: predicting morbidity from sub-clinical findings and clinical proteomics. Injury. 2007;38(3):261-2.

8. Klein MB, Hayden D, Elson C, et al. The association between fluid administration and outcome following major burn: a multicenter study. Ann Surg. 2007;245(4):622-8.

9. Kasotakis G, Sideris A, Yang Y, et al. Aggressive early crystalloid resuscitation adversely affects outcomes in adult blunt trauma patients: an analysis of the Glue Grant database. J Trauma Acute Care Surg. 2013;74(5):1215-21.

10. James MFM, Michell WL, Joubert IA, Nicol AJ, Navsaria PH, Gillespie RS. Resuscitation with hydroxyethyl starch improves renal function and lactate clearance in penetrating trauma in a randomized controlled study: the FIRST trial (Fluids in ResusCitation of Severe Trauma). Br J Anaesth. 2011;107(5):693-702. doi:10. 1093/bja/aer229.

11. Evans JA, van Wessem KJ, McDougall D, et al. Epidemiology of traumatic deaths: comprehensive population-based assessment. World J Surg. 2010;34(1):158-63.

12. Ball CG, Dente CJ, Shaz B, et al. The impact of a massive transfusion protocol $(1: 1: 1)$ on major hepatic injuries: does it increase abdominal wall closure rates? Can J Surg. 2013;56(5):E128-34. doi: $10.1503 /$ cjs.020412. The article describes the implementation of a formal massive transfusion protocol using high plasma and platelet ratios resulted in a substantial increase in abdominal wall approximation following major liver trauma concurrently with a decrease in the delivered volume of crystalloid fluid.

13. CRASH-2 trial collaborators, Shakur H, Roberts I, Bautista R, et al. Effects of tranexamic acid on death, vascular occlusive events, and blood transfusion in trauma patients with significant haemorrhage (CRASH-2): a randomized, placebo-controlled trial. Lancet. 2010;376(9734):23-32. doi:10.1016/S0140-6736(10)60835-5.

14. Thurston B, Chowdhury S, Edu S, Nicol AJ, Navsaria PH. Time since injury is the major factor in preventing tranexamic acid use in the trauma setting: an observational cohort study from a major trauma center in a middle-income country. S Afr J Surg. 2015;53(1):138 . The majority of our bleeding trauma patients in a middle or low-income country do not present within a time frame that allows for safe administration of tranexamic acid therapy.

15. Cotton BA, Gunter OL, Isbell J, et al. Damage control hematology: the impact of a trauma exsanguination protocol on survival and blood product utilization. J Trauma. 2008;64:1177-82.

16. Stinger HK, Spinella PC, Perkins JG, et al. The ratio of fibrinogen to red cells transfused affects survival in casualties receiving massive transfusions at an army combat support hospital. J Trauma. 2008;64:S79-85.

17. O'Keeffe T, Refaai M, Tchorz K, et al. A massive transfusion protocol to decrease blood component use and cost. Arch Surg. 2008;143:686-90.

18. Navsaria P, Thoma M, Nicol A. Foley catheter balloon tamponade for life-threatening hemorrhage in penetrating neck trauma. World $\mathrm{J}$ Surg. 2006;30(7):1265-8.

19. Van Waes OJ, Cheriex KC, Navsaria PH. Management of penetrating neck injuries. Br J Surg. 2012;99 Suppl 1:149-54. doi:10.1002/ bjs.7733. Foley catheter balloon tamponade is useful in patients with penetrating neck injury and active bleeding.

20. Mejia JC, Stewart RM, Cohn SM. Emergency department thoracotomy. Semin Thorac Cardiovasc Surg. 2008;20:13-8.

21. Working Group, Ad Hoc Subcommittee on Outcomes, American College of Surgeons, Committee on Trauma. Practice management guidelines for emergency department thoracotomy. Working Group, Ad Hoc Subcommittee on Outcomes, American College of Surgeons-Committee on Trauma. J Am Coll Surg. 2001;193: 303-9.

22. Lewis G, Knottenbelt JD. Should emergency room thoracotomy be reserved for cases of cardiac tamponade? Injury. 1991;22(1):5-6.

23. Boyd M, Vanek VW, Bourguet CC. Emergency room resuscitative thoracotomy: when is it indicated? J Trauma. 1992;33(5):714-21.

24. Rhee PM, Acosta J, Bridgeman A, et al. Survival after emergency department thoracotomy: review of published data from the past 25 years. J Am Coll Surg. 2000;190(3):288-98.

25. Branney SW, Moore EE, Feldhaus KM, et al. Critical analysis of two decades of experience with postinjury emergency department thoracotomy in a regional trauma center. J Trauma. 1998;45(1):8794.

26. Schwab CW, Adcock OT, Max MH. Emergency department thoracotomy (EDT): a 26-month experience using an "agonal" protocol. Am Surg. 1986;52(1):20-9.

27. Feliciano DV, Bitondo CG, Cruse PA, et al. Liberal use of emergency center thoracotomy. Am J Surg. 1986;152(6):654-9.

28. Velmahos GC, Degiannis E, Souter I, et al. Outcome of a strict policy on emergency department thoracotomies. Arch Surg. 1995;130(7):774-7.

29. Miglietta MA, Robb V, Eachempati SR, et al. Current opinion regarding indications for emergency department thoracotomy. J Trauma. 2001;51:670-6.

30. Rabinovici R, Bugaev N. Resuscitative thoracotomy: an update. Scand J Surg. 2014;103(2):112-9. doi:10.1177/ 1457496913514735. This review demonstrates the indications for resuscitative thoracotomy. The new information is available regarding its use in the pre-hospital urban environment and military settings. It also sheds light on the long-term outcomes, organ donation, and cost and occupational exposure following resuscitative thoracotomy.

31. Hall BL, Buchman TG. A visual, timeline-based display of evidence for emergency thoracotomy. J Trauma. 2005;59:773-7.

32.• Seamon MJ, Haut ER, Van Arendonk K, et al. An evidence-based approach to patient selection for emergency department thoracotomy: a practice management guideline from the Eastern Association for the Surgery of Trauma. J Trauma Acute Care Surg. 2015;79(1): 159-73. doi:10.1097/TA.0000000000000648. A systematic review/guideline developed evidence-based recommendations (Level III). It answered the question: should patients who 
present pulseless after critical injuries (with and without signs of life after penetrating thoracic, extrathoracic, or blunt injuries) undergo emergency department thoracotomy (EDT) (vs. resusCitation without EDT) to improve survival and neurologically intact survival?.

33. Nagy KK, Roberts RR, Smith RF, et al. Trans-mediastinal gunshot wounds: are "stable" patients really stable? World J Surg. 2002;26(10):1247-50.

34. Stassen NA, Lukan JK, Spain DA, et al. Reevaluation of diagnostic procedures for transmediastinal gunshot wounds. J Trauma. 2002;53(4):635-8.

35. Ibirogba S, Nicol AJ, Navsaria PH. Screening helical computed tomographic scanning in haemodynamic stable patients with transmediastinal gunshot wounds. Injury. 2007;38(1):48-52. doi: 10.1016/j.injury.2006.07.039.

36. Bruckner BA, DiBardino DJ, Cumbie TC, et al. Critical evaluation of chest computed tomography scans for blunt descending thoracic aortic injury. Ann Thorac Surg. 2006;81(4):1339-46.

37. Dyer DS, Moore EE, Ilke DN, et al. Thoracic aortic injury: how predictive is mechanism and is chest computed tomography a reliable screening tool? A prospective study of 1,561 patients. J Trauma. 2000;48(4):673-82.

38. Gavant ML. Helical CT grading of traumatic aortic injuries. Impact on clinical guidelines for medical and surgical management. Radiol Clin N Am. 1999;37:553-74. vi.

39. Grossman MD, May AK, Schwab CW, et al. Determining anatomic injury with computed tomography in selected torso gunshot wounds. J Trauma. 1998;45:446-56.

40. Degiannis E, Benn CA, Leandros E, et al. Transmediastinal gunshot injuries. Surgery. 2000;128(1):54-8.

41. Smith MD, Cassidy JM, Souther S, et al. Transesophageal echocardiography in the diagnosis of traumatic rupture of the aorta. N Engl J Med. 1995;332:356-62. doi:10.1056/NEJM199502093320603.

42. Vignon P, Boncoeur MP, François B, et al. Comparison of multiplane transesophageal echocardiography and contrastenhanced helical CT in the diagnosis of blunt traumatic cardiovascular injuries. Anesthesiology. 2001;94(4):615-22.

43. Navid F, Gleason TG. Great vessel and cardiac trauma: diagnostic and management strategies. Semin Thorac Cardiovasc Surg. 2008;20(1):31-8. doi:10.1053/j.semtcvs.2007.11.007.
44. Azizzadeh A, Valdes J, Miller 3rd CC, et al. The utility of intravascular ultrasound compared to angiography in the diagnosis of blunt traumatic aortic injury. J Vasc Surg. 2011;53(3):608-14.

45. Hirshberg A, Mattox KL, Top Knife: The art \& craft of trauma surgery, Chapter 13 Thoracic vascular trauma for the general surgeon 2005;181-197 (Editor Allen MK, tfm Publishing Ltd. Nr Shrewsbury, SY5 6LX, UK)

46. Pate JW, Cole FH, Walker WA, et al. Penetrating injuries of the aortic arch and it branches. Ann Thorac Surg. 1993;55:586.

47.• Yilmaz TH, Evers T, Sussman M, et al. Operating on penetrating trauma to the mediastinal vessels. Scand J Surg. 2013;103:167-74. A comprehensive review on operative techniques of mediastinal vessels.

48. Johnston Jr RH, Wall MJ, Mattox KL. Innominate artery trauma: a thirty-year experience. J Vasc Surg. 1993;17:134.

49. Mattox KL. Fact and fiction about management of aortic transection [editorial]. Ann Thorac Surg. 1989;48:1.

50. Clements RH, Wagmeister LS, Carraway RP. Blunt intrapericardial rupture of the pulmonary artery in a surviving patient. Ann Thorac Surg. 1997;64:258.

51. Petrone P, Asensio JA. Surgical management of penetrating pulmonary injuries. Scand J Trauma Resuscitation Emerg Med. 2009;17: 8. doi:10.1186/1757-7241-17-8.

52. DeBakey ME, Simeone FA. Battle injuries of arteries in World War II: an analysis of 2471 cases. Ann Surg. 1946;123:534.

53. Wall Jr MJ, Mattox KL, DeBakey ME. Injuries to the azygous venous system. J Trauma. 2006;60:357.

54. Demetriades D, Velmahos GC, Scalea TM, et al. Blunt traumatic thoracic aorta injuries: early or delayed repair-results of an American Association for the Surgery of Trauma prospective study. J Trauma. 2009;66:967-73.

55. Demetriades D, Velmahos GC, Scalea TM, et al. Diagnosis and treatment of blunt thoracic aortic injuries: changing perspectives. J Trauma. 2008;64:1415-9.

56. Demetriades D, Velmahos GC, Scalea TM, et al. Operative repair or endovascular stent graft in blunt traumatic thoracic aortic injuries: results of an American Association for the Surgery of Trauma multicenter study. J Trauma. 2008;64:561-71.

57. Forbes TL, Harris JR, Lawlor K, et al. Aortic dilation after endovascular repair of blunt traumatic thoracic aortic injuries. $\mathrm{J}$ Vasc Surg. 2010;52:45-8. 